\title{
Trend and factors associated with iron
} supplementation during pregnancy among women who had pregnancy in the preceding five years of the 2005, 2011 and 2016 EDHS: Multivariate decomposition analysis

Abiyu Abadi Tareke ( $\sim$ abiyu20010@gmail.com )

West Armachiho district health office

Ermias Bekele

Mettu university department of health informatics

Research Article

Keywords: trend, EDHS, Iron supplementation during pregnancy, multivariate decomposition analysis, Ethiopia

Posted Date: June 14th, 2021

DOI: https://doi.org/10.21203/rs.3.rs-596119/v2

License: (a) (i) This work is licensed under a Creative Commons Attribution 4.0 International License. Read Full License 


\section{Abstract}

Introduction: Study estimated in 2019 iron deficiency related anemia accounts $1.89 \%$ and $1.07 \%$ of total disability adjusted life years (DALYS) to reproductive age women globally and sub saran Africa respectively. Anemia at the time of pregnancy may cause small sized baby, preterm birth, and perinatal, neonatal and maternal mortality. Iron and folic acid supplementation during pregnancy will minimize the risk of iron deficiency and risk of anemia.

Methods: for this time series analysis, dataset of 2005, 2011 and 2016 Ethiopian demographic health surveys was used. A total weighted sample of 7234 in 2005, 7786 in 2011 and 7543 in 2016 are encompassed in this study. The Logit based Multivariate decomposition analysis for non-linear response outcome was calibrated to detect the factors contributed to the change in iron supplementation between 2005 and 2016 study period. The Logit based multivariate decomposition analysis utilizes the output from the logistic regression model to assign the observed change in iron supplementation over time into two components. Stata version 16.0 was used to analysis the data.

Results: proportion of iron supplementation during pregnancy increased by more than a third $(32 \%)$ between the period of 2005 and 2016. From the output of multivariate decomposition regression, major of the change in iron supplementation was due to change in coefficients. Women's age, working s status and region of living (all except Dire Dawa) were variables as sources of change in iron supplementation under the category of change in coefficients. Additionally, age, region, wealth status, employment status/working status, ANC follow up to the last pregnancy and media exposure were variables attributable to the change of iron supplementation during pregnancy under the category of endowment.

Conclusion: rate of iron supplementation at the time of pregnancy increased dramatically over the last decade in Ethiopia. Change in the proportion of women aged $\geq 35$ years, population of each region, wealth status (poorer, middle \& richer), working women, ANC follow up and media exposed as well as change in behavior of women aged $\geq 35$ years, employed women and region of living were sources of change for iron supplementation between 2005 and 2016 period.

Keywords: iron supplementation, trends, EDHS, Ethiopia, multivariate decomposition analysis

\section{Background}

Anemia is persisting as a giant health obstacle in the world. According Study estimated in 2019 iron deficiency related anemia accounts $1.89 \%$ and $1.07 \%$ of total disability adjusted life years (DALYS) to reproductive age women globally and sub saran Africa respectively $(1,2)$. In spite of dramatic progress in maternal health programs in the last decades, anemia remains standing health problems in middle and low income countries $(3,4)$. As per World Health Organization (WHO) statement, more than $40 \%$ of pregnant women are suffering from anemia globally and more than fifty percent of anemia was attributable to iron deficiency (2). 
Anemia at the time of pregnancy may cause small sized baby, preterm birth, and perinatal, neonatal and maternal mortality $(5,6)$. Iron and folic acid supplementation during pregnancy will minimize the risk of iron deficiency and risk of anemia(2). To overcome these problems, WHO recommended any pregnant women to take $30 \mathrm{mg}$ to $60 \mathrm{mg}$ of iron and $0.4 \mathrm{mg}$ of folic acid supplementation daily through the mouth $(2,7)$.

Many studies tried to identify factors that determine iron supplementation during pregnancy among those: maternal age(8-10), educational status (11), the poorest wealth index(10,11), frequency of ANC visits $(8,9$, $11)$, family size (8), birth order, timing of ANC initiation $(12,13)$, perceived distance to health facility (12), higher wealth status $(9)$, anemia $(13,14)$ and gravidity $(13)$ were variables that affect iron supplementation status during pregnancy.

In Ethiopia, the performance of iron supplementation at the time of pregnancy is not satisfactory and has not reached the World Health Organization recommendations and evidence about trend of iron supplementation and contributing factors to change of iron supplementation over time are limited. This study aimed to assess the trend and factors associated with change of iron supplementation during pregnancy in Ethiopia using 2005, 2011 and 2016 demographic and health survey data. Findings from this study may support policymakers and program planner through providing information for evidence based decision making and wise utilization of scarce resources. In addition, key insights may get about the effect of characteristics and population structure changes for the improvement of iron supplementation during pregnancy period over the last 11 years.

\section{Methods And Study Setting}

Ethiopia is one of the sub-saran countries and the second largest in populated in Africa. The fourth Ethiopian health sector development plan (HSDP IV) introduced the current three tiered health system. Based on the 2007 Population and Housing Census (PHC) projection estimate a total population of $103,000,000$ in 2021 . According to the 2007 census a majority of the population (83.6\%) was living in rural areas, the average household size was 4.7 persons and 47\% of the total women were between 15 and 49 years of age(15). Primary healthcare (health posts, health centers, and primary hospitals) is delivered at the primary level, serving up to 100,000 population; secondary level services are offered by general hospitals; and tertiary level services are given by specialized hospitals(16). This study used the data of 2016 Ethiopian demographic health survey (EDHS), which was researched from January 18, 2016, to June $27,2016$.

\section{Variables}

\section{Variables of the study}

For this study, iron supplementation was an outcome variable of interest. Women who supplemented with iron while they are pregnant were coded as " 1 " and those not iron supplemented were coded as " 0 ". 
The potential independent variables after deep literature review considered for this study were; Maternal age, Maternal and partner Education, Marital status, Maternal occupation, Wealth status, perceived distance to a health facility, Desirability of last pregnancy, Birth order, media exposure and Autonomy to decide on health care, ANC visits, place of residency, religion, region.

\section{Operational definition}

Media exposure: Created by combining whether a respondent reads the newspaper, listen to the radio, and watch television and labeled as "exposed" and coded " 1 ". If the subject was exposed to at least one of the three Medias labeled as "not exposed" and coded as" 0 ".

Autonomy to decide on health care.: women who decide their health issue alone are labeled as " autonomous" and coded as "1", who decides jointly with their partner were labeled as "jointly" and coded as "2", while those responded their partner alone decides on their health issue were labeled as "un autonomous" and coded as " 0 ".

Working status: women who are categorized under "not working" were coded " 0 " and those who categorized as professional/technical/managerial, clerical, sales, agricultural, services, skilled manual, unskilled manual or others were considered as "working" and coded " 1 ".

\section{Sample Size and Sampling Procedure}

A two staged stratified clustered sampling method was used from all 11 geographic areas (9 regions and 2 city administration). The first strata included a total of 540 EAs in 2005, 624 in 2011 and 645 EAs were selected proportionally to EA size of nine geographical regions and two administrative cities. In the second strata each eleven administrative division was further sub grouped into urban and rural, bringing results of 21 sampling strata. At this moment, by means of equal probability strategy, 27 to 32 households were selected from each cluster. If women had more than one birth history within the last five years preceding the survey, the most recent births were considered for this study. Ultimately, a total weighted sample of 7234 in 2005, 7786 in 2011 and 7543 in 2016 are encompassed in this study. (17-19) (Fig 1)

\section{Data management and analyses}

To adjust for the complex sampling scheme of the demographic health surveys, to restore the representativeness of the survey and to get reliable statistical estimates, data were weighted and "svyset" STATA command was applied in all descriptive and analytical part of analysis. Svyset command was defined as "svyset as svyset [pw=wgt], psu (v021) strata (v022)". All statistical testing was performed at a 95 percent level of significance.

\section{Logit based multivariate decomposition analysis}

To distinguish factors that contributes change of iron supplementation rate from 2005 to 2016, multivariate decomposition analysis for non-linear response outcome was calibrated. The Logit based multivariate decomposition analysis utilizes the output from the logistic regression model to assign the 
observed change in iron supplementation rate between 2005 and 2016 through subdividing into components (i.e. due to endowment and coefficients). Mvdcmp STATA command was applied to identify factors that contributes for change of iron supplementation rate between 2005 and 2016. The change over time (between 2005 and 2016 EDHS) can be explained by the compositional difference between surveys (i.e. differences in characteristics) and/or the difference in effects of explanatory variables (i.e. differences in the coefficients) between the two groups (i.e. 2005 EDHS and 2016EDHS).

For logistic regression, the Logit or log-odd of difference between 2005 and 2016 EDHS is taken as:

Logit (2005)-Logit (2016) $=F(X 2005 * 2016)-(F$ X2005*32016)

$=[(F(X 2005 * \beta 2016)-F(X 2005 \star \beta 2016) / E)]+[(F(X 2005 * \beta 2016)-F(X 2005 * \beta 2016) / C)](20)$

X indicates outcome variable i.e. iron supplementation in this circumstance

Beta ( $\beta$ ) indicates that, regression coefficient of each selected independent variables

The E component refers to change in iron supplementation over the 11 years imputable to differences in endowments or characteristics. The $\mathrm{C}$ component refers to change in iron supplementation over the 11 years imputable to differences in coefficients or effects. Percent contribution with a $95 \%$ confidence interval $(\mathrm{Cl})$ of coefficients and a $\mathrm{p}$-value $<0.05$ were reported.

\section{Ethical consideration}

Approval to download 2016 EDHS dataset was gained from measure DHS program website i.e. http://www.dhsprogram.com through explaining the objective of this study. As this study used district-level aggregated data, informed consent was not obtained from the study participants.

\section{Results}

Table 1 below illustrates Percentage distribution of nominated characteristics of respondent's for 2005, 2011 and 2016 Ethiopian Demographic and Health Surveys separately. It is noticeable that the largest proportion of 25-34 years of age group was noted in all study periods. Highest decline of Percentage of women aged between 15 and 24 years was detected between 2005 and 2016 (i.e. decreased by 2.5\%). Point percent of uneducated women was reduced by 15\% and 12\% from 2005 to 2016 and from 2011 to 2016 respectively. In contrast, percent of primary schooled women were considerably escalated from 2005 to 2011 (by 12\%) and from 2005 to 2016 (by 11.8\%).

Concerning the religion distribution, number of orthodox Christian was the dominant category across the three study period and highest fall was noticed between 2005 and 2016 (by 7\%). Between the period of 2005 and 2016 percentage of Muslim and protestant followers was upraised by $2.6 \%$ and $4.6 \%$ respectively. Additionally, percentage of women from poorest and poorer household's categories remains stable across the three study periods. Conversely, composition of urban residency raised by $6.34 \%$ and $4 \%$ from 2005 to 2011 and from 2005 to 2016 EDHS respectively. 
From 2005 to 2011 time period saw almost quarter decrease in percentage of women who are not working and some increment (by 9\%) was also noted between 2011 and 2016 time period. The percentage of women who perceived the distance from health facilities is not a big problem was elevated from $27 \%$ in 2005 to $42 \%$ in 2016 . However, point percentage of women who had exposed to media was decreased nearly by $3 \%$ percent within the last 11 years of study periods.

Table 1. Percentage distribution of socio-demographic characteristics among respondents using evidence from, 2005, 2011 and 2016 EDHS 


\begin{tabular}{|c|c|c|c|c|c|c|}
\hline Characteristics & $\begin{array}{l}2005 \\
N=7,234\end{array}$ & $\begin{array}{l}2011 \\
N=7786\end{array}$ & $\begin{array}{l}2016 \\
N=7545\end{array}$ & $\begin{array}{l}2011- \\
2005\end{array}$ & $\begin{array}{l}2016- \\
2011\end{array}$ & $2016-2005$ \\
\hline \multicolumn{7}{|l|}{ Respondent's age } \\
\hline $15-24$ & $26.23 \%$ & $25.55 \%$ & $23.77 \%$ & $-0.68 \%$ & $-1.78 \%$ & $-2.46 \%$ \\
\hline $25-34$ & $46.31 \%$ & $49.15 \%$ & $50.38 \%$ & $2.84 \%$ & $1.23 \%$ & $4.07 \%$ \\
\hline $35+$ & $27.46 \%$ & $25.31 \%$ & $25.85 \%$ & $-2.15 \%$ & $0.54 \%$ & $-1.61 \%$ \\
\hline \multicolumn{7}{|l|}{ Region } \\
\hline Tigray & $6.59 \%$ & $6.40 \%$ & $7.05 \%$ & $-0.19 \%$ & $0.65 \%$ & $0.46 \%$ \\
\hline Afar & $0.93 \%$ & $0.99 \%$ & $0.94 \%$ & $0.06 \%$ & $-0.05 \%$ & $0.01 \%$ \\
\hline Amhara & $25.42 \%$ & $24.64 \%$ & $21.52 \%$ & $-0.78 \%$ & $-3.12 \%$ & $-3.90 \%$ \\
\hline Oromia & $37.27 \%$ & $40.16 \%$ & $41.22 \%$ & $2.89 \%$ & $1.06 \%$ & $3.95 \%$ \\
\hline Somali & $3.92 \%$ & $2.51 \%$ & $3.54 \%$ & $-1.41 \%$ & $1.03 \%$ & $-0.38 \%$ \\
\hline Benishangul-gumuz & $0.92 \%$ & $1.19 \%$ & $1.06 \%$ & $0.27 \%$ & $-0.13 \%$ & $0.14 \%$ \\
\hline SNNP & $22.33 \%$ & $20.85 \%$ & $21.13 \%$ & $-1.48 \%$ & $0.28 \%$ & $-1.20 \%$ \\
\hline Gambela & $0.31 \%$ & $0.37 \%$ & $0.27 \%$ & $0.06 \%$ & $-0.10 \%$ & $-0.04 \%$ \\
\hline Harari & $0.20 \%$ & $0.24 \%$ & $0.23 \%$ & $0.04 \%$ & $-0.01 \%$ & $0.03 \%$ \\
\hline Addis Ababa & $1.76 \%$ & $2.33 \%$ & $2.60 \%$ & $0.57 \%$ & $0.27 \%$ & $0.84 \%$ \\
\hline Dire-Dawa & $0.34 \%$ & $0.32 \%$ & $0.44 \%$ & $-0.02 \%$ & $0.12 \%$ & $0.10 \%$ \\
\hline
\end{tabular}

Respondent's educational status

\begin{tabular}{|l|r|r|r|r|r|r|}
\hline No Education & $78.45 \%$ & $66.59 \%$ & $63.11 \%$ & $-11.86 \%$ & $-3.48 \%$ & \multicolumn{1}{|c|}{$-15.34 \%$} \\
\hline Primary & $16.51 \%$ & $28.74 \%$ & $28.32 \%$ & $12.23 \%$ & $-0.42 \%$ & $11.81 \%$ \\
\hline $\begin{array}{l}\text { Secondary\& } \\
\text { above }\end{array}$ & $5.04 \%$ & $4.67 \%$ & $8.57 \%$ & $-0.37 \%$ & $3.90 \%$ & $3.53 \%$ \\
\hline
\end{tabular}

Religion

\begin{tabular}{|l|r|r|r|r|r|r|}
\hline Orthodox & $44.70 \%$ & $42.09 \%$ & $37.97 \%$ & $-2.61 \%$ & $-4.12 \%$ & $-6.73 \%$ \\
\hline Catholic & $1.04 \%$ & $0.99 \%$ & $0.94 \%$ & $-0.05 \%$ & $-0.05 \%$ & $-0.10 \%$ \\
\hline Protestant & $19.20 \%$ & $22.34 \%$ & $21.80 \%$ & $3.14 \%$ & $-0.54 \%$ & $2.60 \%$ \\
\hline Muslim & $32.56 \%$ & $32.38 \%$ & $37.15 \%$ & $-0.18 \%$ & $4.77 \%$ & $4.59 \%$ \\
\hline Traditional & $1.55 \%$ & $1.05 \%$ & $1.28 \%$ & $-0.50 \%$ & $0.23 \%$ & $-0.27 \%$ \\
\hline & $0.94 \%$ & $1.10 \%$ & $0.85 \%$ & $0.16 \%$ & $-0.25 \%$ & $-0.09 \%$
\end{tabular}


Other

Wealth status

\begin{tabular}{|l|r|r|r|r|r|r|}
\hline Poorest & $20.81 \%$ & $21.92 \%$ & $21.77 \%$ & $1.11 \%$ & $-0.15 \%$ & $0.96 \%$ \\
\hline Poorer & $21.23 \%$ & $21.47 \%$ & $21.74 \%$ & $0.24 \%$ & $0.27 \%$ & $0.51 \%$ \\
\hline Middle & $21.73 \%$ & $20.58 \%$ & $20.95 \%$ & $-1.15 \%$ & $0.37 \%$ & $-0.78 \%$ \\
\hline Richer & $19.84 \%$ & $18.93 \%$ & $18.81 \%$ & $-0.91 \%$ & $-0.12 \%$ & $-1.03 \%$ \\
\hline Richest & $16.39 \%$ & $17.10 \%$ & $16.74 \%$ & $0.71 \%$ & $-0.36 \%$ & $0.35 \%$ \\
\hline
\end{tabular}

Place of residency

\begin{tabular}{|c|c|c|c|c|c|c|}
\hline Urban & $8.67 \%$ & $15.01 \%$ & $12.77 \%$ & $6.34 \%$ & $-2.24 \%$ & $4.10 \%$ \\
\hline Rural & $91.33 \%$ & $84.99 \%$ & $87.23 \%$ & $-6.34 \%$ & $2.24 \%$ & $-4.10 \%$ \\
\hline \multicolumn{7}{|c|}{ Timing of ANC visits } \\
\hline Early & $22.68 \%$ & $26.32 \%$ & $32.75 \%$ & $3.64 \%$ & $6.43 \%$ & $10.07 \%$ \\
\hline delayed & $77.32 \%$ & $73.68 \%$ & $67.25 \%$ & $-3.64 \%$ & $-6.43 \%$ & $-10.07 \%$ \\
\hline \multicolumn{7}{|c|}{ Frequency of ANC visits } \\
\hline $1-3$ & $87.80 \%$ & $80.59 \%$ & $67.91 \%$ & $-7.21 \%$ & $-12.68 \%$ & $-19.89 \%$ \\
\hline $4+$ & $12.20 \%$ & $19.41 \%$ & $32.09 \%$ & $7.21 \%$ & $12.68 \%$ & $19.89 \%$ \\
\hline \multicolumn{7}{|l|}{ Birth order } \\
\hline $1-4$ & $57.64 \%$ & $61.47 \%$ & $60.96 \%$ & $3.83 \%$ & $-0.51 \%$ & $3.32 \%$ \\
\hline $5-9$ & $37.43 \%$ & $34.34 \%$ & $36.03 \%$ & $-3.09 \%$ & $1.69 \%$ & $-1.40 \%$ \\
\hline $10+$ & $4.93 \%$ & $4.20 \%$ & $3.02 \%$ & $-0.73 \%$ & $-1.18 \%$ & $-1.91 \%$ \\
\hline \multicolumn{7}{|l|}{ Working status } \\
\hline Not working & $69.00 \%$ & $44.84 \%$ & $53.70 \%$ & $-24.16 \%$ & $8.86 \%$ & $-15.30 \%$ \\
\hline Working & $31.00 \%$ & $55.16 \%$ & $46.30 \%$ & $24.16 \%$ & $-8.86 \%$ & $15.30 \%$ \\
\hline \multicolumn{7}{|c|}{ Perceived distance to health facility } \\
\hline Big problem & $73.32 \%$ & $72.90 \%$ & $58.01 \%$ & $-0.42 \%$ & $-14.89 \%$ & $-15.31 \%$ \\
\hline Not big problem & $26.68 \%$ & $26.99 \%$ & $41.99 \%$ & $0.31 \%$ & $15.00 \%$ & $15.31 \%$ \\
\hline \multicolumn{7}{|c|}{ Autonomy to decision making } \\
\hline Autonomous & $35.81 \%$ & $26.58 \%$ & $19.66 \%$ & $-9.23 \%$ & $-6.92 \%$ & $-16.15 \%$ \\
\hline Jointly & $50.55 \%$ & $61.27 \%$ & $66.50 \%$ & $10.72 \%$ & $5.23 \%$ & $15.95 \%$ \\
\hline Autonomous & $13.42 \%$ & $12.03 \%$ & $13.85 \%$ & $-1.39 \%$ & $1.82 \%$ & $0.43 \%$ \\
\hline \multicolumn{7}{|l|}{ Media Exposure } \\
\hline Not exposed & $62.54 \%$ & $40.10 \%$ & $65.42 \%$ & $-22.44 \%$ & $25.32 \%$ & $2.88 \%$ \\
\hline exposed & $37.46 \%$ & $59.90 \%$ & $34.58 \%$ & $22.44 \%$ & $-25.32 \%$ & $-2.88 \%$ \\
\hline
\end{tabular}




\begin{tabular}{|l|r|r|r|r|r|r|}
\hline$<5$ & $44.64 \%$ & $48.01 \%$ & $46.24 \%$ & $3.37 \%$ & $-1.77 \%$ & $1.60 \%$ \\
\hline $5+$ & $55.36 \%$ & $51.99 \%$ & $53.76 \%$ & $-3.37 \%$ & $1.77 \%$ & $-1.60 \%$ \\
\hline
\end{tabular}

\section{Trend of iron supplementation during pregnancy}

According to table 2 and Fig 2 the prevalence of iron supplementation during pregnancy substantially raised almost by third from 2005 (10.45\%) to 2016 (42.14\%). The second largest improvement of iron supplementation was identified in between study period of 2011 and 2016 (rise by quarter). Generally a significant change (non-over lapping 95\% confidence interval) of iron supplementation during pregnancy was seen alongside the three study period i.e. 2005 to 2011, 2011 to 2016 and 2005 to 2016 EDHS.

From 2005 to 2016 time period; Amhara (by 9.35\%), Oromia (by 8.6\%) and SNNP (by 6\%) were regions with largest amount of improvement in proportion of iron supplementation during pregnancy time. But, minimal rise of proportion of iron supplementation during pregnancy time was noticed from regions; BenishangulGumuz, Harari and Gambela, afar and Diredawa. Tigray region was the only region that showed reduction in iron supplementation over the last 11 years.

Between 2005 and 2016 highest increment of iron supplementation rate was noted among orthodox Christian and Muslim followers i.e. $16 \%$ and $9.3 \%$ rise respectively. But, stable rise of iron supplementation was noted among protestant followers between 2005 and 2016 as well as between 2011 and 2016. Over the decade rural resident showed dramatic escalation in iron supplementation rate from $8.7 \%$ in 2005 to $34.4 \%$, slight increment from 2011 to 2016.

In between 2005 and 2011, the point percentage of iron supplementation showed slight fall down (-0.13\%). After that from 2011-2016 the amount of iron supplementation rose slowly by $0.7 \%$. In addition to this, percent of women with focused ANC visits (four and above number of visits) rose sharply by 18.3 point percentage over the three successive study periods. In regard to birth order, proportion of iron supplementation was rose by 23 and 9 point percent among women who had parity of one to four and five to nine respectively. In between 2005 and 2011, Proportion of iron supplementation was fall down steadily among women with no exposure to media and rapidly rose by $19 \%$ within 5 years of period i.e. 2011-2016. Similar pattern of increment of iron supplementation was also noted among media exposed women between 2005 and 2016. (Table 2)

Table 2: Trends of iron supplementation usage during pregnancy among reproductive age from 2005, 2011 and 2016 EDHS 


\begin{tabular}{|c|c|c|c|c|c|c|}
\hline \multirow[b]{2}{*}{ Characteristics } & \multicolumn{3}{|c|}{$\begin{array}{l}\text { Percentage point In iron } \\
\text { supplementation }\end{array}$} & \multicolumn{3}{|c|}{$\begin{array}{l}\text { Percentage point difference } \\
\text { In iron supplementation }\end{array}$} \\
\hline & $\begin{array}{c}2005 \\
\mathrm{~N}= \\
7,234\end{array}$ & $\begin{array}{c}2011 \\
N=7786\end{array}$ & $\begin{array}{c}2016 \\
N=7545\end{array}$ & $\begin{array}{l}2011 \\
2005\end{array}$ & $\begin{array}{l}2016- \\
2011\end{array}$ & 2016-2005 \\
\hline $\begin{array}{l}\text { Iron } \\
\text { supplemented }\end{array}$ & $10.45 \%$ & $17.35 \%$ & $42.14 \%$ & $6.9 \%$ & $24.79 \%$ & $31.69 \%$ \\
\hline \multicolumn{7}{|c|}{ Respondent's age } \\
\hline $15-24$ & $2.5 \%$ & $15.2 \%$ & $11.1 \%$ & $12.70 \%$ & $-4.10 \%$ & $8.60 \%$ \\
\hline $25-34$ & $4.9 \%$ & $17.7 \%$ & $21.7 \%$ & $12.80 \%$ & $4.00 \%$ & $16.80 \%$ \\
\hline $35+$ & $30.6 \%$ & $17.6 \%$ & $9.3 \%$ & $-13.00 \%$ & $-8.30 \%$ & $-21.30 \%$ \\
\hline \multicolumn{7}{|l|}{ Region } \\
\hline Tigray & $6.60 \%$ & $2.23 \%$ & $5.44 \%$ & $-4.37 \%$ & $3.21 \%$ & $-1.16 \%$ \\
\hline Afar & $0.08 \%$ & $0.23 \%$ & $0.41 \%$ & $0.15 \%$ & $0.18 \%$ & $0.33 \%$ \\
\hline Amhara & $2.16 \%$ & $4.79 \%$ & $11.51 \%$ & $2.63 \%$ & $6.72 \%$ & $9.35 \%$ \\
\hline Oromia & $3.84 \%$ & $4.63 \%$ & $12.41 \%$ & $0.79 \%$ & $7.78 \%$ & $8.57 \%$ \\
\hline Somali & $0.33 \%$ & $0.50 \%$ & $0.98 \%$ & $0.17 \%$ & $0.48 \%$ & $0.65 \%$ \\
\hline $\begin{array}{l}\text { Benishangul- } \\
\text { gumuz }\end{array}$ & $0.07 \%$ & $0.27 \%$ & $0.51 \%$ & $0.20 \%$ & $0.24 \%$ & $0.44 \%$ \\
\hline SNNP & $2.68 \%$ & $3.16 \%$ & $8.75 \%$ & $0.48 \%$ & $5.59 \%$ & $6.07 \%$ \\
\hline Gambela & $0.05 \%$ & $0.11 \%$ & $0.11 \%$ & $0.06 \%$ & $0.00 \%$ & $0.06 \%$ \\
\hline Harari & $0.04 \%$ & $0.08 \%$ & $0.12 \%$ & $0.04 \%$ & $0.04 \%$ & $0.08 \%$ \\
\hline $\begin{array}{l}\text { Addis } \\
\text { Ababa }\end{array}$ & $0.34 \%$ & $0.94 \%$ & $1.66 \%$ & $0.60 \%$ & $0.72 \%$ & $1.32 \%$ \\
\hline Dire-Dawa & $0.04 \%$ & $0.09 \%$ & $0.26 \%$ & $0.05 \%$ & $0.17 \%$ & $0.22 \%$ \\
\hline \multicolumn{7}{|c|}{ Respondent's educational status } \\
\hline $\begin{array}{l}\text { No } \\
\text { Education }\end{array}$ & $2.48 \%$ & $13.00 \%$ & $15.48 \%$ & $2.48 \%$ & $13.00 \%$ & $15.48 \%$ \\
\hline Primary & $3.63 \%$ & $8.06 \%$ & $11.69 \%$ & $3.63 \%$ & $8.06 \%$ & $11.69 \%$ \\
\hline
\end{tabular}




\begin{tabular}{|c|c|c|c|c|c|c|}
\hline $\begin{array}{l}\text { Secondary\& } \\
\text { above }\end{array}$ & $0.48 \%$ & $4.08 \%$ & $4.56 \%$ & $0.48 \%$ & $4.08 \%$ & $4.56 \%$ \\
\hline \multicolumn{7}{|l|}{ Religion } \\
\hline Orthodox & $4.68 \%$ & $8.65 \%$ & $20.56 \%$ & $3.97 \%$ & $11.91 \%$ & $15.88 \%$ \\
\hline Catholic & $0.07 \%$ & $0.12 \%$ & $0.30 \%$ & $0.05 \%$ & $0.18 \%$ & $0.23 \%$ \\
\hline Protestant & $2.18 \%$ & $2.96 \%$ & $8.11 \%$ & $0.78 \%$ & $5.15 \%$ & $5.93 \%$ \\
\hline Muslim & $3.33 \%$ & $5.11 \%$ & $12.58 \%$ & $1.78 \%$ & $7.47 \%$ & $9.25 \%$ \\
\hline Traditional & $0.10 \%$ & $0.06 \%$ & $0.36 \%$ & $-0.04 \%$ & $0.30 \%$ & $0.26 \%$ \\
\hline Other & $0.07 \%$ & $0.14 \%$ & $0.26 \%$ & $0.07 \%$ & $0.12 \%$ & $0.19 \%$ \\
\hline \multicolumn{7}{|l|}{ Wealth status } \\
\hline Poorest & $1.27 \%$ & $2.92 \%$ & $6.83 \%$ & $1.65 \%$ & $3.91 \%$ & $5.56 \%$ \\
\hline Poorer & $1.82 \%$ & $2.63 \%$ & $9.03 \%$ & $0.81 \%$ & $6.40 \%$ & $7.21 \%$ \\
\hline Middle & $2.31 \%$ & $3.17 \%$ & $8.20 \%$ & $0.86 \%$ & $5.03 \%$ & $5.89 \%$ \\
\hline Richer & $2.21 \%$ & $3.40 \%$ & $8.59 \%$ & $1.19 \%$ & $5.19 \%$ & $6.38 \%$ \\
\hline Richest & $2.82 \%$ & $4.92 \%$ & $9.51 \%$ & $2.10 \%$ & $4.59 \%$ & $6.69 \%$ \\
\hline \multicolumn{7}{|c|}{ Place of residency } \\
\hline Urban & $1.73 \%$ & $4.09 \%$ & $7.80 \%$ & $2.36 \%$ & $3.71 \%$ & $6.07 \%$ \\
\hline Rural & $8.71 \%$ & $12.94 \%$ & $34.40 \%$ & $4.23 \%$ & $21.46 \%$ & $25.69 \%$ \\
\hline \multicolumn{7}{|c|}{ Frequency of ANC visits } \\
\hline No & $3.73 \%$ & $3.60 \%$ & $4.30 \%$ & $-0.13 \%$ & $0.70 \%$ & $0.57 \%$ \\
\hline $1-3$ & $3.60 \%$ & $6.08 \%$ & $16.45 \%$ & $2.48 \%$ & $10.37 \%$ & $12.85 \%$ \\
\hline $4+$ & $3.11 \%$ & $7.36 \%$ & $21.43 \%$ & $4.25 \%$ & $14.07 \%$ & $18.32 \%$ \\
\hline \multicolumn{7}{|l|}{ Birth order } \\
\hline $1-4$ & $5.81 \%$ & $10.54 \%$ & $28.64 \%$ & $4.73 \%$ & $18.10 \%$ & $22.83 \%$ \\
\hline $5-9$ & $4.05 \%$ & $5.83 \%$ & $12.78 \%$ & $1.78 \%$ & $6.95 \%$ & $8.73 \%$ \\
\hline $10+$ & $0.57 \%$ & $0.67 \%$ & $0.75 \%$ & $0.10 \%$ & $0.08 \%$ & $0.18 \%$ \\
\hline \multicolumn{7}{|l|}{ Working status } \\
\hline Not & & & & & & \\
\hline working & $7.16 \%$ & $6.93 \%$ & $20.33 \%$ & $-0.23 \%$ & $13.40 \%$ & $13.17 \%$ \\
\hline Working & $3.27 \%$ & $10.10 \%$ & $21.84 \%$ & $6.83 \%$ & $11.74 \%$ & $18.57 \%$ \\
\hline \multicolumn{7}{|c|}{ Perceived distance to health facility } \\
\hline Big problem & $7.21 \%$ & $11.30 \%$ & $20.50 \%$ & $4.09 \%$ & $9.20 \%$ & $13.29 \%$ \\
\hline
\end{tabular}




\begin{tabular}{|l|r|r|r|r|r|r|}
\hline $\begin{array}{l}\text { Not big } \\
\text { problem }\end{array}$ & $3.22 \%$ & $5.73 \%$ & $21.70 \%$ & $2.51 \%$ & $15.97 \%$ & $18.48 \%$ \\
\hline Autonomy to decision making & & & & \\
\hline Autonomous & $3.42 \%$ & $3.28 \%$ & $7.02 \%$ & $-0.14 \%$ & $3.74 \%$ & $3.60 \%$ \\
\hline Jointly & $5.50 \%$ & $11.48 \%$ & $29.05 \%$ & $5.98 \%$ & $17.57 \%$ & $23.55 \%$ \\
\hline Autonomous & $1.34 \%$ & $2.40 \%$ & $6.30 \%$ & $1.06 \%$ & $3.90 \%$ & $4.96 \%$ \\
\hline Media Exposure & & & & & \\
\hline Not exposed & $5.20 \%$ & $5.08 \%$ & $24.20 \%$ & $-0.12 \%$ & $19.12 \%$ & $19.00 \%$ \\
\hline Exposed & $5.22 \%$ & $11.95 \%$ & $17.97 \%$ & $6.73 \%$ & $6.02 \%$ & $12.75 \%$ \\
\hline Ideal number of children & & & & & \\
\hline & $5.60 \%$ & $8.33 \%$ & $20.17 \%$ & $2.73 \%$ & $11.84 \%$ & $14.57 \%$ \\
\hline < 5 & $4.90 \%$ & $8.70 \%$ & $22.00 \%$ & $3.80 \%$ & $13.30 \%$ & $17.10 \%$ \\
\hline $5+$
\end{tabular}

Additionally, there has been similar rose in the prevalence of iron supplementation over the last 11 years in every category of wealth quintile group. Women who desired five and above number of children had risen the proportion of iron supplementation from $4.9 \%$ in 2005 to $22 \%$ in 2016 EDHS.

\section{Regression-based decomposition results}

Table 3 below compares how much of the change of iron supplementation during pregnancy between 2005 and 2016 is imputable to changes in women's characteristics (endowments) and how much to the effects of these characteristics (coefficients). It is apparent that the increment of proportion of iron supplementation was attributable to the coefficient effects of incorporated independent variables. Relatively, the effect of coefficient is more pivotal (coefficient $=0.0718$, representing 78.9 percent share of the total change) than the part explained by the endowment effects (coefficient $=0.2533$; percent shared $=22.08 \%$ ).

Table 3: summary of overall Decomposition Results iron supplementation during pregnancy in Ethiopia 2005 to 2016 EDHS. 


\begin{tabular}{|c|l|l|l|l|}
\hline Iron supplementation & Coefficient & p-value & $95 \%$ CI & Percent \\
\hline E & 0.0718 & 0.000 & {$[0.5722-0.8663]$} & 22.085 \\
\hline C & 0.2533 & 0.000 & {$[0.2304-0.2762]$} & 78.915 \\
\hline R & 0.3251 & 0.000 & {$[0.3065-0.3438]$} & \\
\hline E = difference imputable to Endowment; C difference imputable to coefficients; R= Residual \\
\hline
\end{tabular}

\section{Difference due to characteristics (Endowment)}

Generally, there have been nearly four fifths (78.9\%) change of iron supplementation rate was attributable to change of effect of coefficients between the two study periods (from 2005 to 2016). The multivariate decomposition analysis from table 4 showed that, age, region, wealth status, employment status/working status, ANC follow up to the last pregnancy and media exposure were variables attributable to the change of iron supplementation during pregnancy between 2005 and 2016 in Ethiopia (table 4).

According to table four, the largest proportion of change in iron supplementation (i.e. rise by $16.5 \%$ ) was contributed by compositional differences of women who had ANC follow-up history to their last pregnancy as we go from 2005 to 2016 compared with women who had no ANC follow up history. The compositional differences related to women's working status/employment status was the second most positive contributor to the change of iron supplementation during pregnancy $(+2.62 \%)$, particularly for women who was working/employed compared to women who were unemployed.

Among the very significant contributor compositional factors, positive change of iron supplementation during pregnancy was due to Amhara region $(+1.8 \%)$, SNNP region $(+0.8 \%)$, Somalia region $(+0.45)$ and age greater than 35 years. However, Oromia region contributed to the change of iron supplementation negatively (i.e. decrement by $3.72 \%$ ). Even though statistically significant, compositional factors like wealth status (poorer, middle and rich), media exposure (exposed) and region of living (Addis Ababa, Harari, Gambela, Benishangul and Diredawa)were among the fewer contributors to change of iron supplementation over 11 years of periods.

Table 4: Contribution of selected independent variables to the difference in iron supplementation between 2005 and 2016 in Ethiopia using evidence from EDHS (number of observation=13,594) 


\begin{tabular}{|c|c|c|c|c|}
\hline \multirow[t]{2}{*}{ Variables } & \multicolumn{2}{|c|}{ Difference imputable to Endowment(E) } & \multicolumn{2}{|c|}{ Difference imputable to coefficients(C) } \\
\hline & Coefficients & percent & coefficients & Percent \\
\hline \multicolumn{5}{|l|}{ Age of respondents } \\
\hline $15-24$ & Ref & & ref & \\
\hline $25-34$ & -0.00193 & -0.59331 & -0.01214 & -3.73470 \\
\hline $35+$ & $0.00142 * *$ & $0.43529 * *$ & $-0.02195^{*}$ & $-6.74940 *$ \\
\hline \multicolumn{5}{|c|}{ Educational status of respondents } \\
\hline No Education & Ref & & ref & \\
\hline Primary & 0.00416 & 1.27960 & 0.00577 & 1.77610 \\
\hline Secondary & 0.00097 & 0.29813 & 0.00204 & 0.62849 \\
\hline Higher & 0.00167 & 0.5143 & 0.0003 & 0.091646 \\
\hline \multicolumn{5}{|l|}{ Region } \\
\hline Tigray & Ref & & ref & \\
\hline Afar & $-0.00007 * * *$ & $0.02113^{* * *}$ & $-0.00109 *$ & $-0.33536 *$ \\
\hline Amhara & $0.00588 * * *$ & $1.80720^{* * *}$ & $-0.02120 *$ & $-6.52040 *$ \\
\hline Oromia & $-0.01212 * * *$ & $-3.72670 * * *$ & $-0.10145^{* * *}$ & $-31.20200 * * *$ \\
\hline Somali & $0.00146 * * *$ & $0.44959 * * *$ & $-0.00902^{* * *}$ & $-2.77490 * * *$ \\
\hline Benishangul-gumuz & $-0.00054^{* * *}$ & $-0.16578 * * *$ & $-0.00122^{* *}$ & $-0.37509^{* *}$ \\
\hline SNNP & $0.00263^{* * *}$ & $0.80935^{* * *}$ & $-0.04983^{* * *}$ & $-15.32600 * * *$ \\
\hline Gambela & $0.00015^{* * *}$ & $0.04511^{* * *}$ & $-0.00092^{* * *}$ & $-0.28244 * * *$ \\
\hline Harari & $-0.00008 * * *$ & $0.02361 * * *$ & $-0.00049 * * *$ & $-0.14954^{* * *}$ \\
\hline Addis Ababa & $-0.00307 * * *$ & $-0.94441 * * *$ & $-0.00242 *$ & $-0.74387 *$ \\
\hline Dire-Dawa & $-0.00016 * * *$ & $-0.04812 * * *$ & -0.00017 & 0.05375 \\
\hline \multicolumn{5}{|l|}{ Place of residency } \\
\hline Urban & Ref & & ref & \\
\hline Rural & -0.00036 & -0.11107 & 0.04995 & 15.36100 \\
\hline \multicolumn{5}{|l|}{ Wealth status } \\
\hline Poorest & Ref & & ref & \\
\hline Poorer & $0.00112^{* * *}$ & $0.34494 * * *$ & 0.00124 & 0.38001 \\
\hline Middle & $-0.00061 *$ & $-0.18842^{*}$ & -0.01004 & -3.08750 \\
\hline Richer & $-0.00103^{* *}$ & $-0.31659 * *$ & -0.00798 & -2.45320 \\
\hline Richest & 0.00044 & 0.13626 & -0.00935 & -2.87610 \\
\hline \multicolumn{5}{|l|}{ Religion } \\
\hline Orthodox & Ref & & ref & \\
\hline
\end{tabular}




\begin{tabular}{|c|c|c|c|c|}
\hline Catholic & 0.00017 & 0.05159 & -0.00005 & 0.01686 \\
\hline protestant & -0.00047 & -0.14374 & -0.00388 & -1.19330 \\
\hline Muslin & 0.00011 & 0.03475 & -0.00397 & -1.22180 \\
\hline Traditional & -0.00013 & 0.04123 & 0.00092 & 0.28365 \\
\hline \multicolumn{5}{|l|}{ Working status } \\
\hline Unemployed & Ref & & ref & \\
\hline Employed & $0.00854 * *$ & $2.62680 * *$ & $0.0164 *$ & $5.0568^{*}$ \\
\hline \multicolumn{5}{|l|}{ ANC follow up } \\
\hline No & Ref & & ref & \\
\hline Yes & $0.05375^{* * *}$ & $16.53100 * * *$ & 0.0012 & 0.3728 \\
\hline \multicolumn{5}{|l|}{ Birth order } \\
\hline $1-4$ & Ref & & ref & \\
\hline$\overline{5-9}$ & 0.00002 & 0.00648 & -0.01572 & -4.83570 \\
\hline $10+$ & 0.00218 & 0.67046 & -0.00600 & -1.84560 \\
\hline \multicolumn{5}{|c|}{ Desired number of children } \\
\hline$<5$ & Ref & & ref & \\
\hline$\geq 5$ & 0.00082 & 0.25177 & 0.017232 & 5.2998 \\
\hline \multicolumn{5}{|l|}{ Media exposure } \\
\hline No & Ref & & ref & \\
\hline Yes & $-0.00131 *$ & $-0.40201 *$ & 0.00266 & 0.81738 \\
\hline \multicolumn{5}{|l|}{ Decision making } \\
\hline Not Autonomous & Ref & & ref & \\
\hline Jointly & 0.00225 & 0.69063 & -0.00180 & -0.55256 \\
\hline Autonomous & 0.00002 & 0.00745 & 0.00302 & 0.92858 \\
\hline \multicolumn{5}{|c|}{ Perceived distance from health facility } \\
\hline Big problem & Ref & & ref & \\
\hline Not big problem & 0.00582 & 1.79090 & 0.01029 & 3.16380 \\
\hline Constant & & ficient $=0$. & ercent $=$ & \\
\hline
\end{tabular}

*represents 5 percent, ${ }^{* *}$ represents 10 percent and $* * * 1 \%$ percent level of significance

\section{Changes imputable to effects of coefficients}

Holding the effect of change in compositional factors constant, nearly four fifths changes in iron supplementation during pregnancy was attributable to the difference in effect of coefficient between the two study periods (table 3). In regard to change of iron supplementation attributable to change in coefficients between the two surveys, the most important explanatory variables that provide significant contributions are women's age, working status/employment status and region of living (all except Dire Dawa). The change of women's behavior aged 35 years negatively contributed (decrease by $6.75 \%$ ) to change of iron supplementation. Another interesting contributor was that being employed women increased iron supplementation during pregnancy by 5 point percentage. To illustrate, being employed women increases the possibility of taking iron tablets during pregnancy.

Concerning region of residency, just over a third (31\%) change of iron supplementation was imputable to change behavior of Oromia region population. Change of behavior of population of SNNP, Amhara and 
Somalia regions negatively contributed to the change of iron supplementation during pregnancy between 2005 and 2016 years of study i.e. decrease by $15 \%$ ), $6.5 \%$ and $2.8 \%$ respectively. Negligible contribution were also observed due to change of behavior of population living in Afar, Benishangul-gumuz, Gambela, Harari and Addis Ababa compared to counterpart Tigray region.

\section{Discussion And Conclusion}

Iron supplementation during pregnancy period in Ethiopia rose dramatically between 2005 and 2016 time period. This huge change might reflect the impact of maternal health program initiatives launched by the government of Ethiopia and other stakeholders initiated over the last decades. This study has described the Ethiopian iron supplementation rate during pregnancy and identified factors that have contributed most to the increase in pregnant women iron usage between 2005 and 2016. The study utilized Logit based Multivariate Decomposition for Nonlinear Response Models to decompose the contribution of changes in women's characteristics and changes in effects of these characteristics.

Outputs from the multivariate decomposition regression revealed that the rise in iron supplementation use between 2005 and 2016 is imputable more to the changes in effects of characteristics (coefficients) than to the changes in sample population composition (endowments). Variables such as women's age, working status and region of living (all except Dire Dawa) were the contributing factors under category of change in coefficients of multivariate decomposition. The contribution of change in endowment was due to change (either decrease or increase) in proportion of media exposed women, ANC visits, working women, wealth status (poorer, middle and richer), region of living and women's age (being $\geq 35$ years).

Holding the effect of change in effect of endowment constant, being employed (working) displayed predominant contribution to the rise of iron supplementation. This finding is in line with other studies done in India (21). This can be explained through, employed women may have opportunity to access information related to health in general. Employed women are more likelihood of visits health facilities due to their ability to offer cost of transport and medicine. Other reason might be related to their education level, that educated women are more likely to be hired to job and educated women have better knowledge about the severity and consequence of the problems(14). But, negative relationship was observed between women aged $\geq 35$ years old and iron tablet uptake. This outcome is contrary to other study done in Ethiopia(8), which surmised that as age increases, the likelihood of taking an iron tablet increases. A possible explanation for this might be that sample size and method of analysis difference. As women's age increases the proportion of iron uptake diminishes, this might be due to older women might think of themselves as experienced and confident about their pregnancy outcome and refused to take iron tablets. The more aged, the more likely to have and to manage a large family. This finding, while preliminary, suggests that older pregnant women needs special support related to awareness on iron deficiency related pregnancy outcome. Change in behavior of population residing in Oromia, SNNP and Amhara region decreased iron supplementation rate in a striking way.

From the compositional factors, ANC follow up was the dominant contributor to the change of iron uptake, in which the increment of proportion of women who had at least one ANC visits showed significant impact 
on the elevation of iron tablet uptake. This finding is similar with other studies $(8,9,11,22)$. The possible explanation for this moment is, as iron supplementation is one part of ANC services, women who had exposed to ANC services are more likely to get health information related to iron supplementation and discussion with health care providers about the importance of iron supplementation during pregnancy is inevitable. Additionally, increase in proportion of women who lived Amhara region increases the rate of iron supplementation utilization during pregnancy. However, diminished iron supplementation utilization. Decrease in composition of women who resides in middle and richer households also showed slight fall in prevalence of iron supplementation. Event though, the period of study is quite enough to appreciate significant change of iron supplementation, Even though, the authors tried to analyze change of iron supplementation, due to its secondary nature of the dataset many predictor variables might have missed in this study. (Table 4)

\section{Conclusions}

Over the last 11 years, iron supplementation rate rose significantly among pregnant women in Ethiopia. The Logit based decomposition analysis noted that about $79 \%$ percent of rise of iron supplementation over the period of 2005 to 2016 was attributable to the change of effect of characteristics (coefficients). Variables such as women's age, working status/employment status and region of living (all except Dire Dawa) were the major sources of Change of effect of coefficients. In regard to change of composition of characteristics, age, region, wealth status, employment status/working status, ANC follow up to the last pregnancy and media exposure were the contributory compositional factors. It is better to focus program implementers on rising iron supplementation of Tigray, Harari, Gambela and dire Dawa regions as well as older women.

\section{Abbreviations}

ANC, antenatal care, EDHS, Ethiopian Demographic Health Surveys; EAs, enumeration areas; SNNP, south nations and nationalities people.

\section{Declarations}

\section{Acknowledgments}

We would like to thanks to Ethiopian Central statistical agency for helping to access the dataset for conducting the research and DHS measure program permitting us to download supportive materials.

\section{Author Contributions}

Conceptualization: Abiyu Abadi Tareke, Ermias Bekele Enyew.

Formal analysis: Abiyu Abadi Tareke, Ermias Bekele Enyew.

Investigation: Abiyu Abadi Tareke, Ermias Bekele Enyew. 
Methodology: Abiyu Abadi Tareke, Ermias Bekele Enyew

Resources: Abiyu Abadi Tareke, Ermias Bekele Enyew.

Software: Abiyu Abadi Tareke, Ermias Bekele Enyew

Validation: Abiyu Abadi Tareke, Ermias Bekele Enyew

Visualization: Abiyu Abadi Tareke, Ermias Bekele Enyew

Writing - original draft: Abiyu Abadi Tareke, Ermias Bekele Enyew.

Writing - review \& editing: Abiyu Abadi Tareke, Ermias Bekele Enyew.

Funding: The authors have not received a specific grant for this research from any funding agency in the public, commercial or not-for-profit sectors.

Competing interests: The authors declare no competing interests.

\section{References}

1. Global Burden of Diseases laRFG. anemia,Iron 2019 [cited 2021 may 19]. Available from: https://vizhub.healthdata.org/gbd-compare/.

2. world health organization. e-Library of Evidence for Nutrition Actions (eLENA) 2019 [updated 11 February 2019 cited 2021 may 19]. Available from: https://www.who.int/elena/titles/daily_iron_pregnancy/en/.

3. Stevens GA, Finucane MM, De-Regil LM, Paciorek CJ, Flaxman SR, Branca F, et al. Global, regional, and national trends in haemoglobin concentration and prevalence of total and severe anaemia in children and pregnant and non-pregnant women for 1995-2011: a systematic analysis of populationrepresentative data. The Lancet Global Health. 2013;1(1):e16-e25.

4. Chowdhury AMR, Bhuiya A, Chowdhury ME, Rasheed S, Hussain Z, Chen LC. The Bangladesh paradox: exceptional health achievement despite economic poverty. The Lancet. 2013;382(9906):1734-45.

5. Haider BA, Olofin I, Wang M, Spiegelman D, Ezzati M, Fawzi WW. Anaemia, prenatal iron use, and risk of adverse pregnancy outcomes: systematic review and meta-analysis. Bmj. 2013;346.

6. Rasmussen $\mathrm{KM}$. Is there a causal relationship between iron deficiency or iron-deficiency anemia and weight at birth, length of gestation and perinatal mortality? The Journal of nutrition. 2001;131(2):590S6035 .

7. WHO W. Guideline: daily iron and folic acid supplementation in pregnant women. 2012. 
8. Haile D, Tabar L, Lakew Y. Differences in spatial distributions of iron supplementation use among pregnant women and associated factors in Ethiopia: evidence from the 2011 national population based survey. BMC pregnancy and childbirth. 2017;17(1):1-8.

9. Ba DM, Ssentongo P, Kjerulff KH, Na M, Liu G, Gao X, et al. Adherence to iron supplementation in 22 sub-Saharan African countries and associated factors among pregnant women: a large population-based study. Current developments in nutrition. 2019;3(12):nzz120.

10. Jikamo B, Samuel M. Non-adherence to iron/folate supplementation and associated factors among pregnant women who attending antenatal care visit in selected Public Health Institutions at Hosanna Town, Southern Ethiopia, 2016. J Nutr Disord Ther. 2018;8(230):2161-0509.1000230.

11. Gebremedhin S, Samuel A, Mamo G, Moges T, Assefa T. Coverage, compliance and factors associated with utilization of iron supplementation during pregnancy in eight rural districts of Ethiopia: a cross-sectional study. BMC public Health. 2014;14(1):1-8.

12. Lyoba WB, Mwakatoga JD, Festo C, Mrema J, Elisaria E. Adherence to Iron-Folic Acid Supplementation and Associated Factors among Pregnant Women in Kasulu Communities in NorthWestern Tanzania. International Journal of Reproductive Medicine. 2020;2020:3127245.

13. Gebremariam AD, Tiruneh SA, Abate BA, Engidaw MT, Asnakew DT. Adherence to iron with folic acid supplementation and its associated factors among pregnant women attending antenatal care follow up at Debre Tabor General Hospital, Ethiopia, 2017. PloS one. 2019;14(1):e0210086.

14. Taye B, Abeje G, Mekonen A. Factors associated with compliance of prenatal iron folate supplementation among women in Mecha district, Western Amhara: a cross-sectional study. Pan African Medical Journal. 2015;20(1).

15. Central Statistical Agency. Population Projections for Ethiopia 2007-2037 2013 [cited 2021 march 11]. Available from: https://www.statsethiopia.gov.et/wp-content/uploads/2019/05/ICPS-PopulationProjection-2007-2037-produced-in-2012.pdf.

16. Alebachew A, Waddington C. Improving health system efficiency: Ethiopia: human resources for health reforms. World Health Organization, 2015.

17. Central Statistical Agency - CSA/Ethiopia, ICF. Ethiopia Demographic and Health Survey 2016. Addis Ababa, Ethiopia: CSA and ICF, 2017.

18. Central Statistical Agency/Ethiopia, ORC Macro. Ethiopia Demographic and Health Survey 2005. Addis Ababa, Ethiopia: Central Statistical Agency/Ethiopia and ORC Macro, 2006.

19. Central Statistical Agency/Ethiopia, ICF International. Ethiopia Demographic and Health Survey 2011. Addis Ababa, Ethiopia: Central Statistical Agency/Ethiopia and ICF International, 2012. 
20. Powers DA, Yoshioka H, Yun M-S. mvdcmp: Multivariate decomposition for nonlinear response models. The Stata Journal. 2011;11(4):556-76.

21. Khan REA, Raza MA. Maternal health care: the case of iron supplementation in India. 2013.

22. Lacerte $\mathrm{P}$, Pradipasen $\mathrm{M}$, Temcharoen $\mathrm{P}$, Imamee $\mathrm{N}$, Vorapongsathorn $\mathrm{T}$. Determinants of adherence to iron/folate supplementation during pregnancy in two provinces in Cambodia. Asia Pacific Journal of Public Health. 2011;23(3):315-23.

\section{Figures}

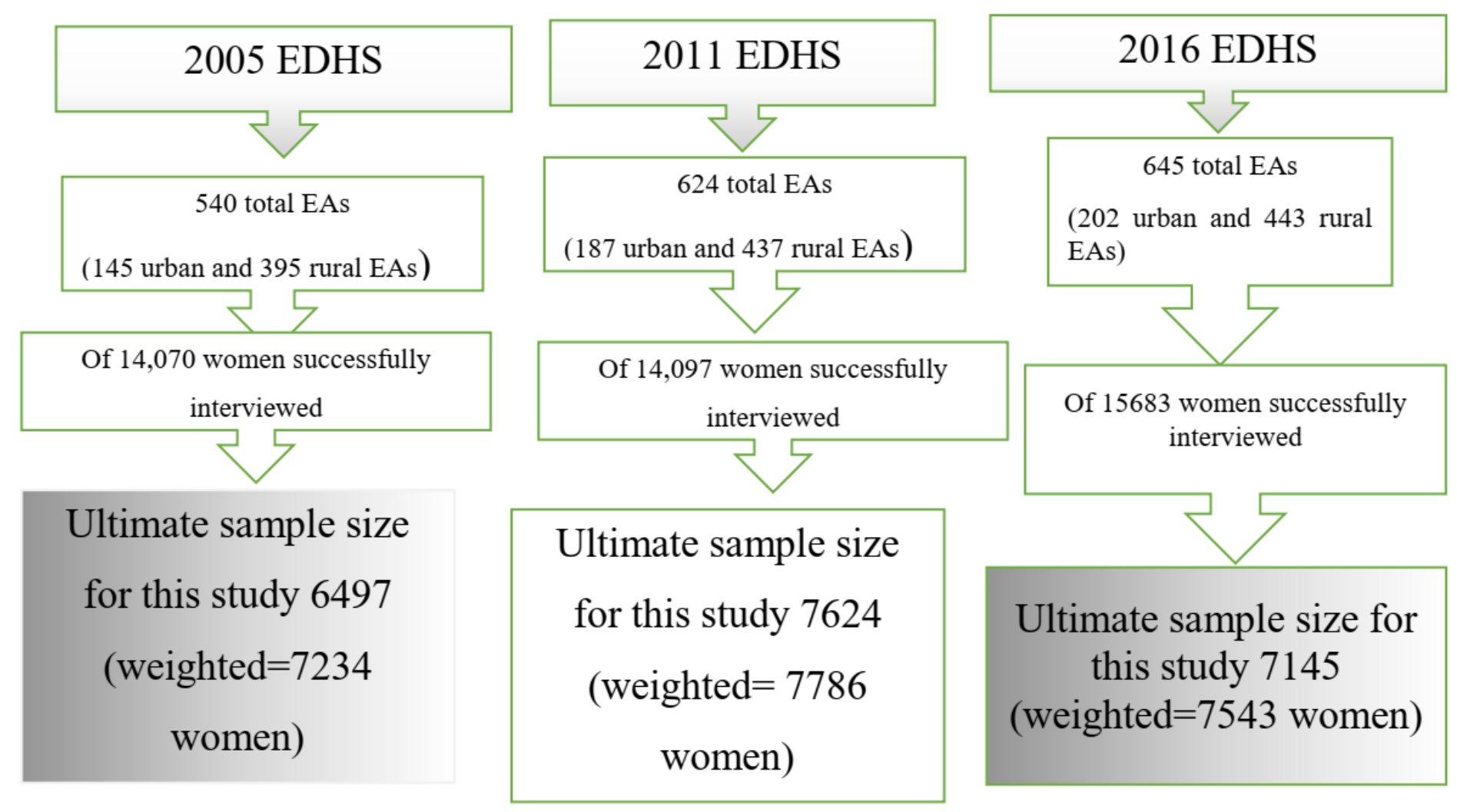

Figure 1

Sampling and sample size determination procedure of 2005, 2011 and 2016 EDHS. 


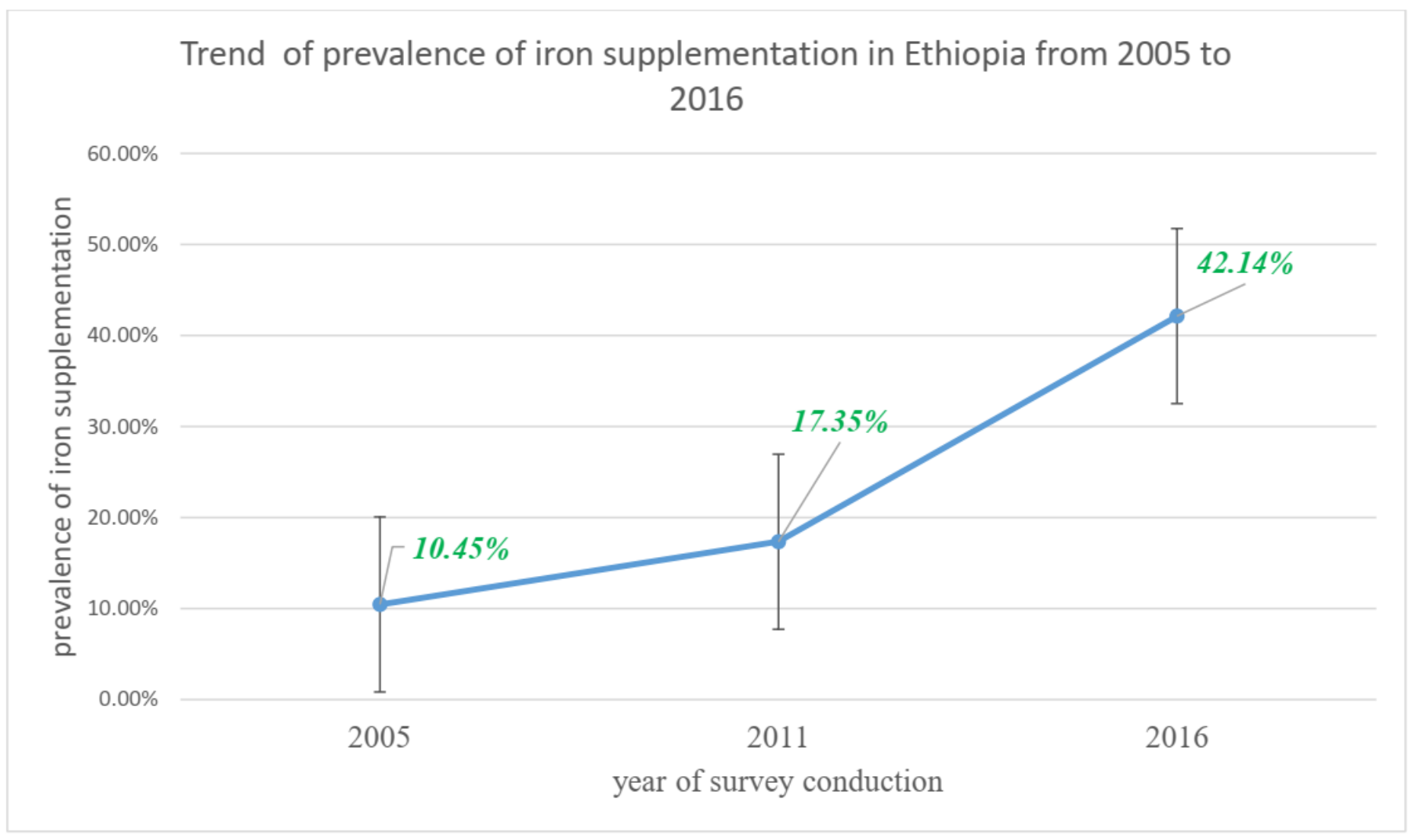

Figure 2

Trends of prevalence of iron supplementation during pregnancy from 2005 to 2016 . 